\title{
Respiratory antibiotic use and Clostridium difficile infection: is it the drugs or is it the doctors?
}

\author{
M H Wilcox
}

The 1990s have seen Clostridium difficile infection (CDI) rise to prominence (fig 1). ${ }^{1-3}$ Why has this occurred? Proposed explanations include spread of virulent strains, increased patient carriage of $C$ difficile, and suboptimal hospital cleaning leading to increased environmental contamination by clostridial spores. ${ }^{1}$ However, given the inextricable link between antibiotic exposure and CDI, increased antimicrobial prescribing in general and/or use of high risk agents must be considered as the main impetus for the rising reports of CDI. The association between cephalosporin use-notably second and, particularly, third generation agents such as cefuroxime and cefotaxime- and CDI is well described. ${ }^{4-9}$ British Thoracic Society (BTS) guidelines for the treatment of community acquired pneumonia (CAP) were published in $1993^{10}$ and recommended the use of second or third generation cephalosporins for severe cases. It has been suggested that these guidelines were responsible for increased cephalosporin prescribing and, as a result, fuelled the increased incidence of CDI. ${ }^{5} 811{ }^{12}$ However, as shown in fig 2, cephalosporin sales to British hospitals have been relatively stable in recent years, ${ }^{13}$ although such analyses may mask prescribing patterns in patients at high risk of CDI, particularly the

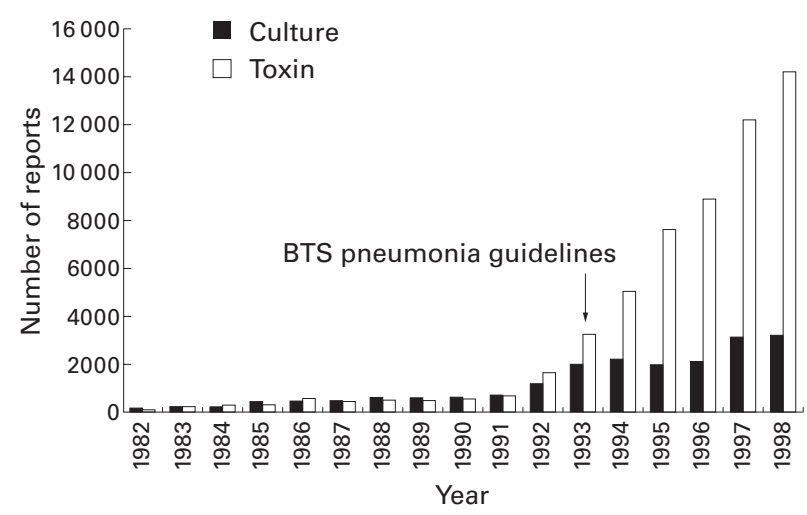

Figure 1 Laboratory reports of $C$ difficile to Communicable Disease Surveillance Centre (England and Wales) 1982-98.

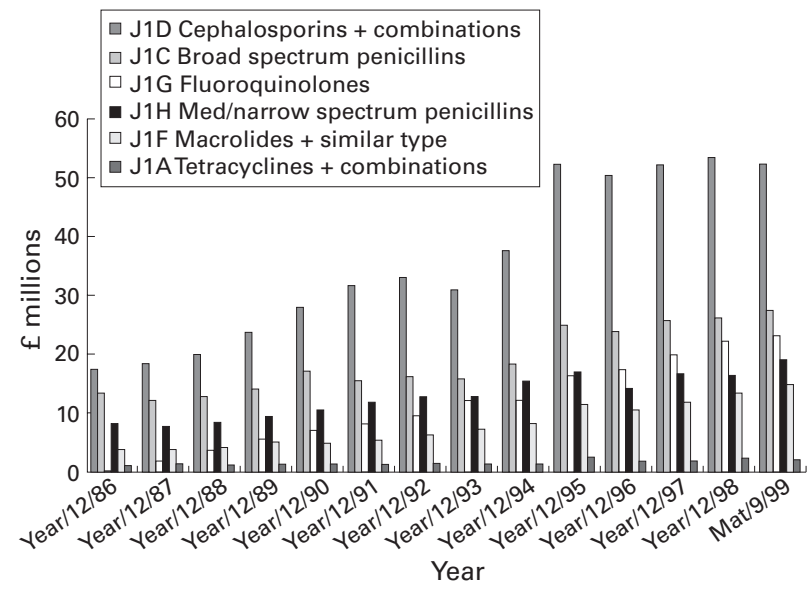

Figure 2 Antibiotic sales into UK hospitals. (Note: a change in data collection occurred between 1994 and 1995 resulting in an increase in recorded antibiotic sales.) elderly. Furthermore, reports of CDI were already increasing before the publication of the BTS guidelines on CAP (fig 1). Nevertheless, individual units have reported increased cephalosporin prescribing (at the very least) coincident with the 1993 guidelines. ${ }^{11}$ Such observations may reflect inappropriate interpretation and/or implementation of the guidelines - for example, overclassification of CAP cases as "severe" rather than "mild/moderate".

Ortqvist reported that there are wide variations in European countries in both general practice and hospital based antibiotic prescribing for CAP. ${ }^{14}$ For example, in 1992 benzylpenicillin and erythromycin were used for hospital treatment of $53 \%$ and $7 \%$, respectively, of cases of CAP in Sweden compared with $17 \%$ and $33 \%$ of cases in the UK. Such variation is not explainable by differences in factors such as antibiotic susceptibility or aetiology of infection. Instead, historical prescribing habits and marketing pressures are probably more influential in determining prescribing practice. Furthermore, there is no good evidence that such variability influences infection outcome, despite higher drug acquisition $\operatorname{costs}^{15}$ and possible complications such as $\mathrm{CDI}^{16}$ which may further increase treatment expense. ${ }^{17}$

It should be noted that many of the data on the propensity of different antimicrobial agents to induce CDI are based on retrospective observations. Such studies rarely control for potentially important confounding variables such as patient age, patient location, and strain endemicity. Accepting these shortcomings, three classes of antimicrobial agents are consistently poorly associated with $C$ difficile infection-namely, aminoglycosides, first generation fluoroquinolones such as ciprofloxacin, and ureidopenicillins \pm $\beta$-lactamase inhibitors (table 1). The only published prospective comparative (ward crossover) study of antibiotics found a significant increased risk (relative risk 7.1) of CDI following empirical treatment with cefotaxime compared with piperacillin-tazobactam. ${ }^{7}$ Other perceived low risk agents include penicillin, trimethoprim, rifampicin, fusidic acid, and nitrofurantoin. It is notable that there are very few published reports associating coamoxiclav with CDI, which is all the more remarkable given the widespread usage of this antimicrobial combination agent. It is difficult to rectify these findings with the widely held theory that CDI results from overgrowth of toxin producing bacteria, secondary to antibiotic mediated impairment of the anaerobic component of the gut florathat is, impaired colonisation resistance. ${ }^{18}$ Simplistically, broad spectrum, particularly anti-anaerobic, agents should be those most associated with CDI. However, in vivo anti-

Table 1 Antibiotics and risk of $C$ difficile infection

\begin{tabular}{lll}
\hline High risk & Medium risk & Low risk \\
\hline Cephalosporins & Ampicillin/amoxycillin & Aminoglycosides \\
Clindamycin & Co-trimoxazole & Metronidazole \\
& Macrolides & Anti-pseudomonal penicillins \\
& Tetracyclines & $\pm \beta$-lactamase inhibitor \\
& & Quinolones \\
& & Rifampicin \\
& & Vancomycin \\
\hline
\end{tabular}

These subdivisions may be prone to bias because of the relative frequency of use of agents and the complexity of interaction between antibiotic exposure, $C$ difficile, and other factors. 
microbial activity, and therefore probably risk of CDI, can be markedly influenced by factors such as drug penetration into the large gut lumen, specific and non-specific antibiotic binding, and gut $\mathrm{pH}$ and redox potential.

Medicines Control Agency data on reports of antibiotic associated colitis in the UK have not been published since $1994 .{ }^{19}$ These crude data were not adjusted for number of prescriptions. Two interesting observations on this league table can be made. Firstly, cefotaxime is somewhat surprisingly not listed, but this may be because the list is biased to those agents that are available in oral formulations (and hence are more frequently prescribed). Despite this latter point, it is notable that co-amoxiclav is not listed. Two recent UK reports have produced data on the comparative risks of antibiotics to induce CDI. ${ }^{56}$ Using locally collected data, after correction for antibiotic combinations, only cefotaxime, ceftriaxone, cefuroxime and ceftazidime were significantly associated with $C$ difficile diarrhoea. $^{5}$ Bignardi carried out a meta-analysis of published studies on antibiotics associated with CDI. ${ }^{6}$ However, the risk ranking of antibiotics produced by this process was undermined by wide $95 \%$ confidence intervals reflecting the wide variation in observed risk of individual antibiotics. This problem reflects the many co-factors which may influence the risk of $C$ difficile infection.

New generation fluoroquinolones have increased activity against Gram positive cocci and often also have marked anti-anaerobe activity. ${ }^{20}$ Enhanced activity against $C$ difficile suggests that agents such as moxifloxacin and gatifloxacin are unlikely to be associated with $C$ difficile infection. However, clinical use of these antibiotics in elderly hospitalised patients where $C$ difficile is endemic requires further study, particularly as the epidemic UK $C$ difficile clone has reduced susceptibility to both old and new fluoroquinolones. ${ }^{21}$

To consider the risk of an antimicrobial agent to induce CDI in an exposed patient alone may be an oversimplification. In reality, patient exposure to multiple antibiotics is common and multiple antibiotics are frequently used in the " $C$ difficile health care setting". Starr et $a l^{12}$ proposed a theoretical model based on the loosely termed concept of "herd (ward) immunity", partly to explain the observation that patients with symptomatic CDI have not uncommonly been exposed to a relatively low risk antimicrobial agent. Essentially, patients in a ward can be considered to be in one of four $C$ difficile risk categories based on whether they are susceptible or resistant to colonisation and if they are colonised or non-colonised. High risk antibiotics, as well as inducing CDI directly, may also make patients more susceptible to infection (directly) by virtue of effects on gut flora and (indirectly) by spread of strains between patients via the ward environment. Subsequent exposure to low risk antibiotics may then be sufficient to induce CDI in patients primed (directly or indirectly) by other agents.

The spectre of antibiotic resistance, particularly the increasing prevalence of penicillin resistant pneumococci, ${ }^{22}$ and the experience of CDI should see significant changes in future antibiotic prescribing for respiratory tract sepsis. Evidence for the lack of effectiveness of antibiotics in conditions such as type 1 chronic bronchitis should be translated into less profligate antimicrobial prescribing. ${ }^{23}$ In general, there is likely to be a shift away from cephalosporin therapy, especially in the elderly, and, indeed, several UK units, prompted by concerns about CDI, have reported on such changes. ${ }^{81124}$ Imminent revised BTS guidelines for the management of CAP are likely to move towards recent US guidelines which recommend alternatives to $\beta$-lactam therapy including newer generation fluoroquinolones with enhanced Gram positive (pneumococcal) activity, macrolides, and tetracycline. ${ }^{25}$
Such recommendations are aimed at mild to moderate cases of CAP and guidelines continue to advocate second or third generation cephalosporins for severe episodes of infection. It will therefore be important to ensure that an over-conservative approach to treatment resulting in unnecessary use of broad spectrum antibiotics does not occur. Validation of the effectiveness of guidelines should be encouraged, ${ }^{15}$ particularly when the evidence base for such recommendations was not robust. There will probably be pressure to increase the proportion of patients with CAP treated as outpatients using validated guidelines. ${ }^{26}{ }^{27}$ Antibiotic treatment outside the hospital setting will reduce the risk of CDI because of a lower prevalence of $C$ difficile, particularly epidemic strains. A projected increase of approximately 1.5 million in the elderly population in England and Wales in the next 20 years will inevitably mean more patients at risk of CDI. ${ }^{28}$ Infection treatment guidelines should consider the issue of antibiotic prescribing in the elderly and, if necessary, make specific recommendations which minimise the risk of CDI. "First do no harm" is an important starting point for all those who prescribe antibiotics.

M H WILCOX

Department of Microbiology,

The General Infirmary and University of Leeds,

Old Medical School, Leeds LS1 3EX, UK

email:markwi@pathology.leeds.ac.uk

1 Wilcox MH. Cleaning up Clostridium difficile infection. Lancet 1996;348:767-8. 2 Clostridium difficile in England and Wales: weeks 27-53/98. Communicable Disease Report 1999;9:59.

Wilcox the UK, 1993-1996. F Hosp Infect 1998;39:181-7.

4 Riley TV. Clostridium difficile: a pathogen of the nineties. Eur $\mathcal{F}$ Clin Microbiol Infect Dis 1998;17:137-41.

5 Zadik PM, Moore AP. Antimicrobial associations of an outbreak of diarrhoea due to Clostridium difficile. $\mathcal{F}$ Hosp Infect 1998;39:189-93.

6 Bignardi GE. Risk factors for Clostridium difficile infection. F Hosp Infect 1998; 40:1-15.

Settle CD, Wilcox MH, Fawley WN, et al. Prospective study of the risk of Clostridium difficile diarrhoea in elderly patients following treatment with cefotaxime or piperacillin-tazobactam. Alimentary Pharmacol Ther 1998;12: $1217-23$

8 McNulty C, Logan M, Donald IP, et al. Successful control of Clostridium difficile infection in an elderly care unit through use of a restrictive antibiotic policy. F Antimicrob Chemother 1997;40:707-1

Jones EM, Kirkpatrick BL, Feeny R, et al. Hospital-acquired Clostridium difficile diarrhoea. Lancet 1997;349:1176-7.

10 British Thoracic Society. Guidelines for the management of community acquired pneumonia in adults admitted to hospital. Br $\mathcal{f}$ Hosp Med 1993;49:346-50.

11 Impallomeni M, Galletly MP, Wort SJ, et al. Increased risk of diarrhoea caused by Clostridium difficile in elderly patients receiving cefotaxime. BMF 1995;311: 1345-6.

12 Starr J M, Rogers T R, Impallomeni M. Hospital-acquired Clostridium difficile diarrhoea and herd immunity. Lancet 1997;349:426-8.

13 Intercontinental Medical Statistics (IMS). Medical Data Index. 4th quarter 1998

14 Ortqvist A. Antibiotic treatment of community acquired pneumonia in clinical

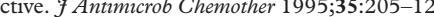

15 Gleason PP, Kapoor WM, Stone RA, et al. Medical outcomes and antimicrobial costs of the use of the American Thoracic Society guidelines for outpatients with community acquired pneumonia. $\mathcal{F} A M A$ 1997;278:32-9.

16 MacGowan AP, Feeney R, Brown I, et al. Health care resource utilization and antimicrobial use in elderly patients with community-acquired lower respiratory tract infection who develop Clostridium difficile-associated diarrhoea. $\mathcal{F}$ Antimicrob Chemother 1997;39:537-41.

17 Wilcox MH, Cunniffe JG, Trundle C, et al. Financial burden of hospitalacquired Clostridum difficile infection. F Hosp Infect 1996;34:23-30.

18 Borriello SP, Barclay FE. An in vitro model of colonisation resistance to Clostridium difficile infection. F Med Microbiol 1986;21:299-309.

19 Medicines Control Agency. Antibiotic-associated colitis. Curr Probl Pharmacovigilance 1994;20:7.

20 Edlund C, Sabouri S, Nord CE. Comparative in vitro activity of BAY 12-8039 and five other antimicrobil Microbiol Infect Dis 1998;17:193-5.

21 Wilcox MH, Fawley W, Freeman J, et al. In vitro activity of new generation fluoroquinolones against genotypically distinct and indistinguishable Clostridium difficile isolates. F Antimicrob Chemother 2000 (submitted).

22 Forward KR. The epidemiology of penicillin resistance in Streptococcus pneumoniae. Semin Respir Infect 1999;14:243-54.

23 Anthonisen NR, Manfreda J, Warren CP, et al. Antibiotic therapy in exacerbations of chronic obstructive pulmonary disease. Ann Intern Med 1987;106: 196-204.

24 Ludlam H, Brown N, Sule O, et al. An antibiotic policy is associated with reduced risk of Clostridum difficile-associated diarrhoea. Age Ageing 1999;28: 578-80.

25 Bartlett JG, Brieman RS, Mandell LA. Community acquired pneumonia in adults: guidelines for management. Clin Infect Dis 1998;26:811-38.

26 Fine MJ, Auble TE, Yealy DM, et al. A prediction rule to identify low risk patients with community acquired pneumonia. N Engl f Med 1997;336:243-50.

27 Atlas SJ, Benzer TI, Borowsky LH, et al. Safely increasing the proportion of Atlas SJ, Benzer TI, Borowsky LH, et al. Safely increasing the proportion of
patients with community acquired pneumonia treated as out-patients. Arch Intern Med 1998:158:1350-6.

28 Population Trends 51 and Monitor PP2 91/1, London. 